Proceedings of the

International Geometry Center

Vol. 13, no. 4 (2020) pp. 115-124

\title{
On the de Rham cohomology of locally trivial Lie groupoids over triangulated manifolds
}

\author{
J. R. Oliveira
}

\begin{abstract}
Based on the isomorphism between Lie algebroid cohomology and piecewise smooth cohomology of a transitive Lie algebroid, it is proved that the de Rham cohomology of a locally trivial Lie groupoid $G$ on a smooth manifold $M$ is isomorphic to the piecewise de Rham cohomology of $G$, in which $G$ and $M$ are manifolds without boundary and $M$ is smoothly triangulated by a finite simplicial complex $K$ such that, for each simplex $\Delta$ of $K$, the inverse images of $\Delta$ by the source and target mappings of $G$ are transverses submanifolds in the ambient space $G$. As a consequence, it is shown that the piecewise de Rham cohomology of $G$ does not depend on the triangulation of the base.
\end{abstract}

Анотація. На основі ізоморфізму між когомологіями алгеброїдів Лі та кусково-гладкими когомологіями транзитивних алгеброїдів Лі доведено, що когомології де Рама локально тривіального групоїда Лі $G$ на гладкому многовиді $M$ ізоморфні кусковим когомологіям де Рама $G$, за умови, що $G$ і $M$ - це многовиди без межі, причому $M$ допускає гладку триангуляцію до скінченного симпліциального комплексу $K$ такого, що для кожного симплексу $\Delta$ з $K$ прообраз $\Delta$ відносно початкового та кінцевого відображення $G$ є трансверсальними підмноговидами в $G$. В якості наслідку, показано, що кускові когомології де Рама $G$ не залежать від триангуляції бази.

The author is partially supported by MICINN, Grant MTM2014-56950-P. 2010 Mathematics Subject Classification: 55N35, 57T99, 58H99

Keywords: de Rham cohomology of Lie groupoids, Lie algebroid cohomology

Ключові слова: Когомології де Рама групоїдів Лі, когомології Лі алгеброїдів DOI: http://dx.doi.org/10.15673/tmgc.v13i4.1753 


\section{INTRODUCTION}

It is well known that the de Rham theorem states that the integration mapping over smooth singular chains on a smooth manifold induces an isomorphism between the de Rham cohomology and the singular cohomology of that manifold (see [15]).

When a manifold is smoothly triangulated by a simplicial complex, the notion of differential form can be defined on the corresponding simplicial space as being a family of differential forms defined on the simplices of the simplicial complex which agree on the intersection of two simplices and satisfy a regular property when restricted to each simplex. A differential can be defined by the corresponding exterior derivative on each simplex, yielding a cochain algebra. Sullivan in [14] and Whitney in [17] show that the cohomology of that cochain algebra is isomorphic to classic cohomologies of the space.

The de Rham-Sullivan theorem (see Sullivan [14, Theorem 7.1]) implies that the restriction mapping from the cochain algebra of the smooth forms on the manifold to the cochain algebra of the piecewise smooth forms on the simplicial space induces an isomorphism in cohomology.

Mishchenko and Oliveira in [11] have extended similar constructions considered by Whitney and Sullivan to transitive Lie algebroids defined over triangulated manifolds in which the regularity property of differential forms is the smoothness. They proved that the restriction mapping induces an isomorphism in cohomology (for details, see [11]).

Since Lie algebroids are the infinitesimal objects of Lie groupoids, the present work is an extension of the Mishchenko-Oliveira's work to Lie groupoids defined over triangulated manifolds. In order to clarify better the intrinsic nature of this extension, fix a locally trivial Lie groupoid $G$ on a closed smooth manifold $M$, in which $G$ is also without boundary, and a triangulation of $M$ such that the inverse images of each simplex $\Delta$ by the source and target mappings of $G$ are transverses submanifolds in $G$. Since $G$ is locally trivial, its Lie algebroid is transitive and the Lie algebroid of the restriction of $G$ to a simplex coincides with the Lie algebroid of $G$ restricted to that simplex. Likewise to the notion of piecewise smooth forms on Lie algebroids defined by Mishchenko-Oliveira in [11], an algebra of invariant piecewise smooth forms can be defined on $G$. As shown in [4] or in [16], the de Rham algebra made of all invariant smooth forms on $G$ is isomorphic to the algebra of all smooth forms of its Lie algebroid. This isomorphism jointly with the isomorphism given in Mishchenko-Oliveira's theorem ([11, Theorem 5.1]) will ensure that the algebra made of all invariant piecewise smooth forms on $G$ is quasi-isomorphic to the algebra 
of all piecewise smooth forms of its Lie algebroid. We will use this quasiisomorphism and [11, Corollary 5.5] to show that the piecewise de Rham cohomology of $G$ does not depend on the triangulation of $M$.

Acknowledgments. I want to thank to Aleksandr Mishchenko, Jesus Alvarez, James Stasheff and Nicolae Teleman for their strong dynamism to discuss several topics concerning this work. I also am sincerely grateful to the referee for many comments and suggestions which led to an improvement of the paper.

\section{De Rham Cohomology of Lie groupoids}

We begin by reviewing briefly basic definitions and constructions concerning Lie groupoids and Lie algebroids. An extensive discussion on these issues can be found in the book [10] by Mackenzie. The papers $[3,5-9,12,18]$ contain a detailed exposition and many examples. Various definitions and properties stated through the entire paper can be found, at level of cell spaces, in $[1,13,14,17]$. Given a smooth manifold $M$ and an integer $p \geqslant 0$, it is well known that the subset of $M$ consisting of all points $x \in M$ such that the pairing $(M, x)$ is locally diffeomorphic to a sectors of index $p$ is a submanifold of $M$, called the boundary of index $p$ of $M$. Throughout this paper, we shall work on manifolds which are smooth, finite-dimensional and possibly with boundaries of different indices.

Lie groupoids. Let $G$ and $M$ be two smooth manifolds. The manifold $G$ is called a Lie groupoid with base $M$ if the following is given: two surjective submersions $\alpha: G \rightarrow M$ and $\beta: G \rightarrow M$, called the source projection and the target projection respectively, a smooth mapping $1: M \rightarrow G$ called the object inclusion mapping, a smooth multiplication

$$
G * G=\{(h, g) \in G \times G: \alpha(h)=\beta(g)\} \rightarrow G
$$

and a mapping $G \rightarrow G$ denoted by $g \mapsto g^{-1}$ and called inverse mapping, satisfying the identities shown in Definitions 1.1.1 and 1.1.3 of Mackenzie's book [10].

The smooth mapping

$$
(\beta, \alpha): G \rightarrow M \times M, \quad g \mapsto(\beta(g), \alpha(g)),
$$

is called the anchor of the Lie groupoid $G$ with base $M$. The Lie groupoid $G$ is said to be locally trivial if its anchor is a surjective submersion. For each $x, z \in M$, the manifolds $G_{x}=\alpha^{-1}(x)$ and $G^{z}=\beta^{-1}(z)$ are called the $\alpha$-fibre of $\mathrm{G}$ in $x$ and the $\beta$-fibre of $\mathrm{G}$ in $z$ respectively. Analogously, if $X$ and $Z$ are subsets of $M$, the sets $\alpha^{-1}(X)$ and $\beta^{-1}(Z)$ of $G$ are denoted by $G_{X}$ and $G^{Z}$ respectively. 
We assume now that $G$ and $M$ are manifolds without boundary. If $X$ and $Z$ are submanifolds of $M$, possibly with boundaries of different indices, then the sets $G_{X}$ and $G^{Z}$ are submanifolds of $G$. Let $N$ be a submanifold of $M$, possibly with boundaries of different indices, such that the manifolds $G_{N}$ and $G^{N}$ are transverses submanifolds in the ambient space $G$. By taking the restrictions of the projections and of the object inclusion mapping, the manifold $G_{N} \cap G^{N}$ is a Lie groupoid with base $N$. The Lie groupoid $G_{N} \cap G^{N}$ will be called the Lie groupoid restriction of $G$ to $N$ and denoted by $G_{N}^{! !}$. The Lie groupoid $G_{N}^{! !}$coincide with the pullback Lie groupoid of $G$ over the inclusion $i: N \hookrightarrow M$ (see the sections 1.5 and 2.3 of [10] for more details on restrictions of Lie groupoids).

Lie algebroids. Let $M$ be a smooth manifold, $T M$ the tangent bundle to $M$ and $\Gamma(T M)$ the Lie algebra of the vector fields on $M$. A Lie algebroid on $M$ is a vector bundle $\pi: \mathcal{A} \rightarrow M$ with base $M$ equipped with a vector bundle morphism $\rho: \mathcal{A} \rightarrow T M$, called anchor of $\mathcal{A}$, and a structure of real Lie algebra on the vector space $\Gamma(\mathcal{A})$ of the sections of $\mathcal{A}$ such that the mapping $\rho_{\Gamma}: \Gamma(\mathcal{A}) \rightarrow \Gamma(T M)$, induced by $\rho$, is a Lie algebra homomorphism and the action of the algebra $\mathcal{C}^{\infty}(M)$ on $\Gamma(\mathcal{A})$ satisfies the natural condition:

$$
[\xi, f \eta]=f[\xi, \eta]+\left(\rho_{\Gamma}(\xi) \cdot f\right) \eta
$$

for each $\xi, \eta \in \Gamma(\mathcal{A})$ and $f \in \mathcal{C}^{\infty}(M)$. The Lie algebroid $\mathcal{A}$ is called transitive if the anchor $\gamma$ is surjective.

Let $\varphi: N \hookrightarrow M$ be a submanifold, possibly with boundaries of different indices and assume that $\mathcal{A}$ is transitive. We recall that the Lie algebroid restriction of $\mathcal{A}$ to the submanifold $N$, denoted by $\mathcal{A}_{N}^{! !}$, is the Lie algebroid $\varphi ! ! \mathcal{A}$ as being the pullback of $\mathcal{A}$ by the mapping $\varphi$ (for more details, see $[3$, $10,11])$.

From Lie groupoids to Lie algebroids. We recall the construction of the Lie algebroid of a Lie groupoid. Let $M$ be a smooth manifold and $G$ a Lie groupoid on $M$ with source projection $\alpha: G \rightarrow M$ and target projection $\beta: G \rightarrow M$. Denote by $1: M \rightarrow G$ the object inclusion mapping of $G$ and $G_{x}=\alpha^{-1}(x)$ the $\alpha$-fibre of $\mathrm{G}$ in $x$, for each $x \in M$. Denote by $\mathcal{A}(G)$ the disjoint union $\bigsqcup_{x \in M} T_{1_{x}} G_{x}$ equipped with the structure of vector bundle on $M$. Consider the mapping

$$
\rho: \mathcal{A}(G) \rightarrow T M
$$

defined by $\rho(a)=D \beta_{1_{x}}(a)$. Define a Lie bracket on $\Gamma(\mathcal{A}(G))$ in the following way: for each $\xi$ and $\eta \in \Gamma(\mathcal{A}(G))$, the Lie bracket is defined by

$$
[\xi, \eta]=\left[\xi^{\prime}, \eta^{\prime}\right]_{G}
$$


in which $\xi^{\prime}$ and $\eta^{\prime}$ denote the unique $\alpha$-right-invariant vector fields on $G$ such that $\xi_{1_{x}}^{\prime}=\xi_{x}$ and $\eta_{1_{x}}^{\prime}=\eta_{x}, \forall x \in M$ (see [4]). Then, $(\mathcal{A}(G),[\cdot, \cdot], \rho)$ is a Lie algebroid on $M$ and is called the Lie algebroid of the Lie groupoid $G$.

Lie groupoids and Lie algebroids enjoy various properties of Lie groups and Lie algebras. We notice that not every Lie algebroid is integrable to a Lie groupoid. Theorem 4.1 of [2] shows necessary and sufficient conditions so that a Lie algebroid is integrable to a Lie groupoid.

Proposition 3.5.18 of [10] states that, if the Lie groupoid $G$ is locally trivial, the Lie algebroid $\mathcal{A}(G)$ is transitive.

Next proposition relates the restriction of a locally trivial Lie groupoid with the restriction of its Lie algebroid.

Proposition 2.1. Let $G$ be a locally trivial Lie groupoid on a smooth manifold $M$, in which $G$ and $M$ are manifolds without boundary, and $\mathcal{A}(G)$ denote the Lie algebroid of $G$. Let $N$ be a submanifold of $M$ such that $G_{N}$ and $G^{N}$ are transverses submanifolds in the ambient space $G$. Then, the Lie algebroid of $G_{N}^{! !}$is the Lie algebroid $\mathcal{A}_{N}^{! !}$.

Proposition 2.1 comes directly from the proposition 4.3 .11 of [10], noting that the pullback Lie groupoid of $G$ over the inclusion $i: N \hookrightarrow M$ exists when $G$ is locally trivial (cf. the last paragraph of the page 63 , the proposition 2.3.1 and the first paragraph of the page 65 of [10]).

Smooth forms on Lie groupoids. Let $G$ be a Lie groupoid on a smooth manifold $M, \alpha: G \rightarrow M$ the source projection, $\beta: G \rightarrow M$ the target projection, and 1: $M \rightarrow G$ the object inclusion mapping. Since $\alpha$ is a surjective submersion, it induces a foliation $\mathcal{F}$ on $G$. Let $T \mathcal{F}$ denote the tangent bundle of $\mathcal{F}$ and $\mathbb{R}_{M}$ the trivial vector bundle $M \times \mathbb{R}$ on $M$ of fibre $\mathbb{R}$.

Definition 2.2. A smooth $\alpha$-form of degree $p$ on the Lie groupoid $G$ is a smooth section of the exterior vector bundle $\bigwedge^{p}\left(T^{*} \mathcal{F} ; \mathbb{R}_{M}\right)$.

Therefore, a smooth form on $G$ is a family $\omega=\left(\omega_{g}\right)_{g \in G}$ such that, for each $g \in G$, one has

$$
\omega_{g} \in \bigwedge^{p}\left(T_{g}^{*} G_{\alpha(g)} ; \mathbb{R}\right)
$$

The set $\Omega_{\alpha}^{*}(G ; M)$ of all smooth $\alpha$-forms on $G$ is a commutative graded algebra. The usual exterior derivative along the $\alpha$-fibres is defined by

$$
\left(d_{\alpha}^{p} \omega\right)\left(X_{1}, X_{2}, \ldots, X_{p+1}\right)=\sum_{j=1}^{p+1}(-1)^{j+1} X_{j} \cdot\left(\omega\left(X_{1}, \ldots, \widehat{X}_{j}, \ldots, X_{p+1}\right)\right)+
$$




$$
+\sum_{i<k}(-1)^{i+k} \omega\left(\left[X_{i}, X_{k}\right], X_{1}, \ldots, \widehat{X_{i}}, \ldots, \widehat{X_{k}}, \ldots, X_{p+1}\right)
$$

in which $\omega \in \Omega_{\alpha}^{p}(G ; M)$ and $X_{1}, X_{2}, \ldots, X_{p+1}$ are smooth vector $\alpha$-fields on $G$. The complex $\Omega_{\alpha}^{*}(G ; M)$ is a cochain algebra defined over $\mathbb{R}$.

We are going now to consider invariant forms. For each $g \in G$, the right translation $R_{g}$ corresponding to $g$ is the mapping $R_{g}: G_{\beta(g)} \rightarrow G_{\alpha(g)}$ defined by $R_{g}(h)=h g$. A smooth form $\omega \in \Omega_{\alpha}^{*}(G ; M)$ is called right invariant or simply invariant if the equality

$$
\left(R_{g}\right)^{*}\left(\left(\iota_{\alpha(g)}\right)^{*} \omega\right)=\left(\iota_{\beta(g)}\right)^{*} \omega
$$

holds for each $g \in G$, in which $\iota_{x}: G_{x} \hookrightarrow G$ denotes the inclusion mapping for each $x \in M$ (see the third section of [4] in which the representation is the trivial representation on the trivial bundle $M \times \mathbb{R}$ on $M$ of fibre $\mathbb{R}$ ). The set $\Omega_{\alpha, R}^{*}(G ; M)$, consisting of all $\alpha$-forms on $G$ which are invariant under all groupoid right translations, is a subcomplex of $\left(\Omega_{\alpha}^{*}(G ; M), d_{\alpha}^{*}\right)$ and hence it is a cochain algebra.

Definition 2.3. Keeping the same hypotheses and notations as above, the de Rham cohomology of $G$ is the cohomology vector space of the cochain algebra $\Omega_{\alpha, R}^{*}(G ; M)$. This cohomology vector space is denoted by $H_{\alpha, R}^{*}(G ; M)$.

Smooth forms on Lie algebroids. We shall recall briefly the notion of smooth forms on Lie algebroids and its cohomology. Let $M$ be a smooth manifold and $\mathcal{A}$ a Lie algebroid on $M$. Denote by $\mathbb{R}_{M}$ the trivial vector bundle $M \times \mathbb{R}$ on $M$ of fibre $\mathbb{R}$. A smooth form on $\mathcal{A}$ is a section of $\bigwedge^{*}\left(\mathcal{A}^{*} ; \mathbb{R}_{M}\right)$. The set $\Omega^{*}(\mathcal{A} ; M)$ of all smooth forms on $\mathcal{A}$ is a commutative cochain algebra defined over $\mathbb{R}$, in which the differential

$$
d^{p}: \Omega^{p}(\mathcal{A} ; M) \rightarrow \Omega^{p+1}(\mathcal{A} ; M)
$$

is given by

$$
\begin{aligned}
& d^{p} \omega\left(X_{1}, X_{2}, \ldots, X_{p+1}\right)= \\
& =\sum_{j=1}^{p+1}(-1)^{j+1}\left(\gamma \circ X_{j}\right) \cdot\left(\omega\left(X_{1}, \ldots, \widehat{X}_{j}, \ldots, X_{p+1}\right)\right)+ \\
& \quad+\sum_{i<k}(-1)^{i+k} \omega\left(\left[X_{i}, X_{k}\right], X_{1}, \ldots, \widehat{X}_{i}, \ldots, \widehat{X}_{k}, \ldots, X_{p+1}\right)
\end{aligned}
$$

for $\omega \in \Omega^{p}(\mathcal{A} ; M)$ and $X_{1}, X_{2}, \ldots, X_{p+1} \in \Gamma(\mathcal{A})$.

The Lie algebroid cohomology of $\mathcal{A}$ is the cohomology vector space of the cochain algebra $\Omega^{*}(\mathcal{A} ; M)$. This cohomology vector space is denoted by $H^{*}(\mathcal{A} ; M)$. 
We notice that [4, Proposition 7], combined with the subsequent paragraphs, states that the mapping

$$
\Psi: \Omega_{\alpha, R}^{*}(G ; M) \rightarrow \Omega^{*}(\mathcal{A} ; M), \quad \Psi(\omega)_{x}=\omega_{1_{x}},
$$

is an isomorphism of cochain algebras (cf. the paragraph preceding of [16, Theorem 1.2]). In addition, we have

$$
H_{\alpha, R}^{*}(G ; M) \cong H^{*}(\mathcal{A} ; M)
$$

(cf. [16, Theorem 1.2]).

\section{Piecewise de Rham cohomology of Lie groupoids}

We begin by recalling Mishchenko-Oliveira's theorem (see [11, Theorem 5.1]). Let $M$ be a compact smooth manifold, smoothly triangulated by a simplicial complex $K$, and $\mathcal{A}$ a transitive Lie algebroid on $K$. A piecewise smooth form on $\mathcal{A}$ is a family $\left(\omega_{\Delta}\right)_{\Delta \in K}$ of smooth forms, each form $\omega_{\Delta}$ defined on $\mathcal{A}_{\Delta}^{! !}$, such that, if $\Delta$ and $\Delta^{\prime}$ are simplices of $K$ in which $\Delta^{\prime}$ is a face of $\Delta$,

$$
\left(\omega_{\Delta}\right)_{/ \Delta^{\prime}}=\omega_{\Delta^{\prime}}
$$

Let $\Omega^{*}(\mathcal{A} ; M)$ and $\Omega^{*}(\mathcal{A} ; K)$ denote respectively the cochain algebras of smooth forms and piecewise smooth forms on $\mathcal{A}$. Consider the restriction mapping

$$
\Omega^{*}(\mathcal{A} ; M) \rightarrow \Omega^{*}(\mathcal{A} ; K), \quad \omega \mapsto(\omega / \Delta)_{\Delta \in K}
$$

Mishchenko-Oliveira's theorem states that this mapping induces an isomorphism in cohomology.

We introduce now the notion of piecewise de Rham cohomology of Lie groupoids. Let $G$ be a locally trivial Lie groupoid with base $M$ such that $G$ and $M$ are manifolds without boundary and $M$ is smoothly triangulated by a simplicial complex $K$. Let $\alpha: G \rightarrow M, \beta: G \rightarrow M$ and $1: M \rightarrow G$ denote respectively the source projection, the target projection and the object inclusion mapping. Suppose that, for each simplex $\Delta$ of $K$, the manifolds $G_{\Delta}$ and $G^{\Delta}$ are transverses submanifolds in the ambient space $G$. Analogous to piecewise smooth forms on Lie algebroids, we give now the notion of invariant piecewise form on $G$.

Definition 3.1. An invariant piecewise $\alpha$-form of degree $p$ on $G$ is a family $\omega=\left(\omega_{\Delta}\right)_{\Delta \in K}$ such that, for each simplex $\Delta \in K, \omega_{\Delta} \in \Omega_{\alpha, R}^{p}\left(G_{\Delta}^{! !} ; \Delta\right)$ is an invariant $\alpha$-form of degree $p$ on $G_{\Delta}^{! !}$and, if $\Delta$ and $\Delta^{\prime}$ are two simplices of $K$ in which $\Delta^{\prime}<\Delta$, the equality $\left(\omega_{\Delta}\right) / \Delta^{\prime}=\omega_{\Delta^{\prime}}$ holds.

The $C^{\infty}(G)$-module of all invariant piecewise $\alpha$-forms of degree $p$ on $G$ is denoted by $\Omega_{\alpha, R}^{p}(G ; K)$. A wedge product and an exterior derivative can 
be defined on the module

$$
\Omega_{\alpha, R}^{*}(G ; K)=\bigoplus_{p \geqslant 0} \Omega_{\alpha, R}^{p}(G ; K)
$$

by the corresponding operations on each algebra $\Omega_{\alpha, R}^{*}\left(G_{\Delta}^{! !} ; \Delta\right)$, giving to $\Omega_{\alpha, R}^{*}(G ; K)$ a structure of cochain algebra defined over $\mathbb{R}$.

Definition 3.2. The piecewise de Rham cohomology of the Lie groupoid $G$, denoted by

$$
H_{\alpha, R}^{*}(G ; K),
$$

is the cohomology of the cochain algebra $\Omega_{\alpha, R}^{*}(G ; K)$.

Our aim now is to relate the piecewise de Rham cohomology $H_{\alpha, R}^{*}(G ; K)$ of $G$ to the cohomology $H^{*}(\mathcal{A}(G) ; K)$ of its Lie algebroid $\mathcal{A}(G)$. For that, we have to consider a mapping $\phi$ from the complex $\Omega_{\alpha, R}^{*}(G ; K)$ to the complex $\Omega^{*}(\mathcal{A}(G) ; K)$. In order to obtain such mapping $\phi$, we recall that, for each simplex $\Delta$ of $K$, we have an isomorphism

$$
\psi_{\Delta}: \Omega_{\alpha, R}^{p}\left(G_{\Delta}^{! !} ; \Delta\right) \rightarrow \Omega^{p}\left(\mathcal{A}\left(G_{\Delta}^{! !}\right) ; \Delta\right)
$$

given by $\left(\psi_{\Delta}(\omega)\right)_{x}=\omega_{1_{x}}$. Consider now an invariant piecewise $\alpha$-form

$$
\omega=\left(\omega_{\Delta}\right)_{\Delta \in K} \in \Omega_{\alpha, R}^{p}(G ; K) .
$$

For each simplex $\Delta \in K$, take the smooth form

$$
\xi_{\Delta}=\psi_{\Delta}\left(\omega_{\Delta}\right) \in \Omega^{p}\left(\mathcal{A}\left(G_{\Delta}^{! !}\right) ; \Delta\right) .
$$

If $\Delta^{\prime}$ is a simplex of $K$ such that $\Delta^{\prime}$ is a face of $\Delta$, then $\left(\xi_{\Delta}\right) / \Delta^{\prime}=\xi_{\Delta^{\prime}}$ and so $\xi=\left(\xi_{\Delta}\right)_{\Delta \in K}$ is a piecewise smooth form on $\mathcal{A}(G)$. Keeping these hypotheses and notations, we state our next proposition.

Proposition 3.3. The mapping $\Phi: \Omega_{\alpha, R}^{*}(G ; K) \rightarrow \Omega^{*}(\mathcal{A}(G) ; K)$ defined by $\Phi\left(\left(\omega_{\Delta}\right)_{\Delta \in K}\right)=\left(\psi_{\Delta}\left(\omega_{\Delta}\right)\right)_{\Delta \in K}$ is well defined and is an isomorphism of cochain algebras.

We can state now the main proposition of this paper. Denote by $r_{G}$ the restriction mapping

$$
r_{G}: \Omega_{\alpha, R}^{*}(G ; M) \rightarrow \Omega_{\alpha, R}^{*}(G ; K), \quad r_{G}(\omega)=(\omega / \Delta)_{\Delta \in K},
$$

and assume that $M$ is compact. Our proposition is the following.

Proposition 3.4. The mapping $r_{G}$ induces an isomorphism in cohomology. 
Proof. The diagram

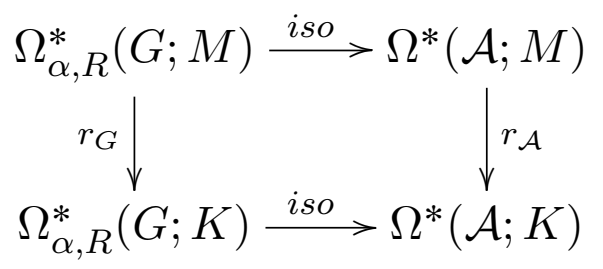

is commutative, where $r_{\mathcal{A}}$ is the restriction mapping given in MishchenkoOliveira's theorem (see [11, Theorem 5.1]). Apply that theorem in cohomology and the proof is done.

Our last proposition states that the piecewise de Rham cohomology of a locally trivial Lie groupoid on a compact triangulated manifold does not depend on the triangulation of the base, that is, for any simplicial subdivision of the simplicial complex, the piecewise de Rham cohomologies of the two triangulated manifolds are isomorphic. Precisely, this statement is our next proposition.

Corollary 3.5. Keeping the same hypotheses and notations as above, let $L$ be other simplicial complex which is a simplicial subdivision of $K$. Then, the piecewise de Rham cohomology of $G$ obtained by the triangulation corresponding to $K$ is isomorphic to the piecewise de Rham cohomology of $G$ obtained by the triangulation corresponding to L. In addition, this isomorphism is induced by the restriction mapping.

Proof. Denote by $\phi: \Omega_{\alpha, R}^{*}(G ; K) \rightarrow \Omega_{\alpha, R}^{*}(G ; L)$ the mapping given by restriction. The diagram

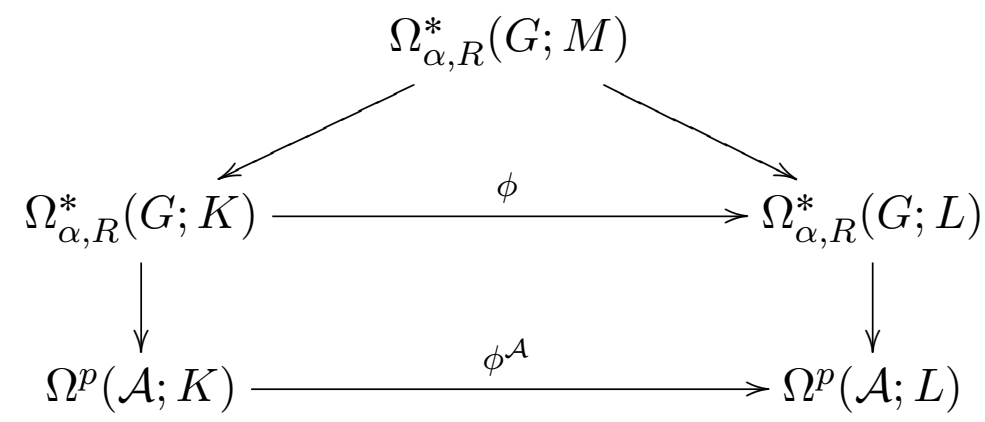

is commutative. By [11, Corollary 5.5], the mapping $\phi^{\mathcal{A}}$ induces an isomorphism in cohomology. Then by propositions 3.3 and 3.4 above, the mappings non labeled induce isomorphisms in cohomology and so the mapping $\phi$ also induces an isomorphism in cohomology.

\section{REFERENCES}

[1] Jesús A. Álvarez López, Manuel Calaza. Witten's perturbation on strata. Asian J. Math., 21(1):47-125, 2017, doi: 10.4310/AJM.2017.v21.n1.a2. 
[2] Marius Crainic, Rui Loja Fernandes. Integrability of Lie brackets. Ann. of Math. (2), 157(2):575-620, 2003, doi: 10.4007/annals.2003.157.575.

[3] Philip J. Higgins, Kirill Mackenzie. Algebraic constructions in the category of Lie algebroids. J. Algebra, 129(1):194-230, 1990, doi: 10.1016/0021-8693(90)90246-K.

[4] Jan Kubarski. Pradines-type groupoids over foliations; cohomology, connections and the Chern-Weil homomorphism. Institute of Mathematics, Technical University of Lodz, 1986, https://im.p.lodz.pl/ kubarski/forum/7Preprint2.pdf.

[5] Jan Kubarski. Pradines-type groupoids. Rend. Circ. Mat. Palermo (2) Suppl., (16):125$143,1987$.

[6] Jan Kubarski. The Chern-Weil homomorphism of regular Lie algebroids. Publications du Département de mathématiques (Lyon), pages 1-69, 1991, www.numdam.org/item/ PDML_1991 1 1_ (in eng).

[7] Syaoyu Li, A. S. Mishchenko. Classification of couplings for transitive Lie algebroids. Dokl. Akad. Nauk, 460(5):517-519, 2015, doi: 10.1134/s1064562415010263.

[8] Xiaoyu Li, A. S. Mishchenko. The existence and classification of couplings between Lie algebra bundles and tangent bundles. Topology Appl., 201:291-308, 2016, doi: $10.1016 /$ j.topol.2015.04.020.

[9] XiaoYu Li, A. S. Mishchenko, V. Gasimov. Erratum to: "Mackenzie obstruction for the existence of a transitive Lie algebroid" [RJMP 21 (4), 544-548 (2014)] [ 3284964]. Russ. J. Math. Phys., 22(1):143, 2015, doi: 10.1134/S106192081501015X.

[10] Kirill C. H. Mackenzie. General theory of Lie groupoids and Lie algebroids, volume 213 of London Mathematical Society Lecture Note Series. Cambridge University Press, Cambridge, 2005, doi: 10.1017/CBO9781107325883.

[11] A. S. Mishchenko, J. R. Oliveira. Whitney-Sullivan constructions for transitive Lie algebroids, to appear.

[12] Jean Pradines. Théorie de Lie pour les groupoïdes différentiables. Relations entre propriétés locales et globales. C. R. Acad. Sci. Paris Sér. A-B, 263:A907-A910, 1966.

[13] D. Sullivan. Differential forms and the topology of manifolds. In Manifolds-Tokyo 1973 (Proc. Internat. Conf., Tokyo, 1973), pages 37-49, 1975.

[14] Dennis Sullivan. Infinitesimal computations in topology. Inst. Hautes Études Sci. Publ. Math., (47):269-331 (1978), 1977, http://www.numdam.org/item?id=PMIHES_ $1977 \_47 \ldots 269 \_0$.

[15] André Weil. Sur les théorèmes de de Rham. Comment. Math. Helv., 26:119-145, 1952, doi: 10.1007/BF02564296.

[16] Alan Weinstein, Ping Xu. Extensions of symplectic groupoids and quantization. $J$. Reine Angew. Math., 417:159-189, 1991.

[17] Hassler Whitney. Geometric integration theory. Princeton University Press, Princeton, N. J., 1957.

[18] L. X. Yu, A. S. Mishchenko, V. Gasimov. Mackenzie obstruction for the existence of a transitive Lie algebroid. Russ. J. Math. Phys., 21(4):544-548, 2014, doi: 10.1134/S1061920814040128.

Received: January 30, 2020, accepted: November 15, 2020.

J. R. Oliveira

Department of Mathematics, University of Minho, Braga, Portugal

Email: jmo@math.uminho.pt 